\title{
, Unraveling the Glyco-Puzzle: Glycan Structure Identification by Capillary Electrophoresis
}

\author{
${ }_{3}$ Stefan Mittermayr, ${ }^{\dagger}$ Jonathan Bones, ${ }^{*}$ and András Guttman, ${ }^{*}, \S$ \\ $4{ }^{\dagger}$ Horváth Laboratory of Bioseparation Sciences, Regional Center for Molecular Medicine, University of Debrecen, Debrecen, Hungary \\ $5{ }^{*}$ NIBRT-The National Institute for Bioprocessing Research and Training, Fosters Avenue, Mount Merrion, Blackrock, Co. Dublin, \\ 6 Ireland \\ $7{ }^{\S}$ MTA-PE Translational Glycomics Group, University of Pannonia, Veszprem, Hungary
}

\begin{abstract}
8 ABSTRACT: State-of-the-art high-resolution separation tech-
9 niques play an important role in the full structural elucidation

10 of glycans. Capillary electrophoresis (CE) offers a rapid yet

11 simple method for exhaustive carbohydrate profiling. CE is a

12 versatile analytical platform, which can be operated in several

13 separation modes, simply by altering separation conditions

14 during operation. For in-depth glycan structural analysis, CE

15 has also gained significantly from the additional resolution

16 introduced by complementary and orthogonal separation

17 techniques such as ion exchange or hydrophilic interaction

18 chromatography. Commercially available mass spectrometry (MS) interfaces have not only brought this information-rich 19 detection technique within reach, but CE also represents an expedient highly efficient separation inlet for MS, capable of 20 separating isobaric oligosacchar $\Omega$ somers prior to MS detection and MS/MS fragmentation based identification. This 21 Perspective gives a sophisticate 2 pression of the versatility of capillary electrophoresis for deep structural elucidation of 22 carbohydrates derived from glycoproteins of biomedical interest. Different separation modes for the analysis of both charged and 23 neutral glycans, such as influencing electroosmotic flow, using complexation/interaction based secondary equilibria, and the use 24 of charged and neutral labels are compared. The merits of introducing orthogonal and complementary techniques, such as 25 exoglycosidase digestion arrays, analytical/preparative chromatography and mass spectrometric detection, and extending the 26 dynamic range and resolution of CE are all thoroughly discussed.
\end{abstract}

${ }_{27} \Gamma$ he structural elucidation of protein derived glycosylation, 28 which may possess vast structural diversity including 29 positional and/or linkage isomerism, poses a substantial 30 analytical challenge. The discovery of different glycan moieties' 31 involvement in protein confirmation or function, cell-ce 32 signaling, and reflection of cellular or even organis 2 33 physiological state has attracted major research attention. 34 Because of the analytical complexity associated with glyco35 sylation analysis, a variety of orthogonal analytical techniques is 36 used, often in combined fashion, and generally including 37 separation driven methods combined with optical, chemical, or 38 mass selective detection. ${ }^{1}$ Capillary electrophoresis (CE) today 39 is an important tool in aveling the glyco-puzzle and 40 represents a rapid yet high 2 olving analytical platform.

41 In the current discerning Perspective of the structural 42 elucidation potential of capillary electrophoresis in analyzing 43 complex protein derived carbohydrates of biomedical and 44 biopharmaceutical interest is presented. Auxiliary techniques, 45 such as sequential exoglycosidase digestion, lectin affinity 46 partitioning, analytical/preparative chromatography and mass 47 spectrometry (MS) detection in conjunction with tandem mass 48 spectrometry (MS/MS) fragmentation are discussed based 49 upon their structural identification capabilities when interfaced 50 with CE.

\section{TECHNICAL OVERVIEW}

51

The technical simplicity of capillary electrophoresis can be 52 deceptive; $\mathrm{CE}$ is a highly adaptive versatile technique, enabling 53 several separation modes simply by altering the separation 54 conditions such as the background electrolyte used. Controlling 55 the electroosmotic flow (EOF) phenomenon, that is the 56 directional bulk flow generated under an electric field by ions 57 attracted to capillary surface charges and its direction (co- or 58 counter-electrophoretic mobility, $\mu_{\mathrm{e}}$ ), allows altering the 59 migration time window and the experimental run time. EOF 60 toward the detection site facilitates a stable liquid flow and CE 61 current for electrospray ionization (ESI) with hyphenated mass 62 spectrometric detection $^{2}$ or can generate a reverse migration 63 order $^{3,4}$ when driving against analyte electrophoretic mobilities 64 (with $\mu_{\mathrm{EOF}}>\mu_{\mathrm{e}}$ ). Suppression of the EOF by covalent or 65 dynamic coating of surface charges can remarkably elevate 66 experimental reproducibility. Under arheic (without flow) 67 conditions, capillary zone electrophoresis (CZE) separates 68 analytes based on their charge to hydrodynamic volume ratio 69 differences thus rendering it a powerful tool for carbohydrate 70

Received: February 26, 2013

Accepted: April 5, 2013 
71 isomer identification. The selectivity of CE for the separation of 72 similar oligosaccharides can be improved by introducing 73 secondary equilibria, such as borate complexation, ${ }^{5}$ micellar 74 surfactants, ${ }^{6-9}$ chromatographic (pseudo-) stationary phases, ${ }^{10}$ 75 or by polymeric additives. 11

76 Protein derived carbohydrates from physiological samples are 77 often only available in limited quantities, lack chromophores or 78 fluorophores, and frequently contain differentially charged 79 species. Therefore, derivatization with charged fluorogenic 80 agents is a popular means to increase detector sensitivity and 81 enable the simultaneous separation of neutral and charged 82 analytes using electric field mediated methods. While optical 83 on-column detection techniques such as laser induced 84 fluorescence (LIF) enable high sensitivity and selectivity they 85 do not however, provide additional glycan structural 86 information. The choice of a labeling agent is strongly 87 dependent on the application and needs to be carefully 88 selected according to the background electrolyte (BGE), EOF, 89 sample characteristics, and also the detection method. 90 Enzymatically released $N$-linked glycans by peptide- $N^{4}-(N$ 91 acetyl- $\beta$-glucosaminyl)asparagine amidase (PNGase F) main92 tain the free amino group from the side chain of the parent 93 asparagine and the resulting glycosylamine can be derivatized 94 with amine reactive dyes under basic conditions. ${ }^{12,13}$ More 95 commonly, the liberated glycosylamines are converted to 96 reducing sugars (aldehyde form) at acidic $\mathrm{pH}$ and reacted 97 with primary amino group containing dyes via reductive 98 amination. ${ }^{14-16}$ However, low $\mathrm{pH}$ and high temperatures can 99 promote analyte hydrolysis and the potential loss of labile 100 glycan constituents as well as selective labeling need to be 101 diligently precluded by optimizing the derivatization reac102 tion. $^{17,18}$ A favorable derivatization agent not only needs to 103 meet reaction chemistry, size, and $\mathrm{pH}$ stable charge state 104 requirements but also has to be compatible with the available 105 detection platforms (e.g., laser excitation wavelength). On106 column complexation, ${ }^{19}$ EOF, or charged residues (e.g., sialic 107 acids) can also provide sufficient separation mobility. Under108 ivatized glycans are disadvantageous for sensitive optical 109 detection but are commonly applied in mass spectrometric 110 analysis. ${ }^{20}$ A free reducing end enhances MS fragmentation 111 options but may also increase analyte complexity due to 112 anomericity. ${ }^{21}$ Conjugated glycans potentially reach higher 113 ionization yields. ${ }^{15,22}$

114 The transition to online mass detection techniques with 115 electrospray ionization brings about the necessity to alter $\mathrm{CE}$ 116 separation conditions, including replacement of outlet buffer 117 reservoir and associated electrical circuit closing as well as 118 introducing volatile background electrolytes, to achieve MS 119 compatibility. Key for successful hyphenation of CE and MS are 120 interfaces that produce a stable spray with low flow (preferably $121<20 \mathrm{~nL} / \mathrm{min}$ ) and allow nearly independent optimization of the 122 separation and ionization sections. Although, a certain degree of 123 compromise needs to be accepted: popular sheath flow 124 interfaces use a sheath liquid at the capillary outlet, which 125 can be optimized for stable ionization but at the cost of 126 sensitivity due to sample dilution. Direct or sheathless 127 connections generally incorporate low flow rates, high 128 sensitivity, and reduced ion suppression, but a common BGE 129 for reasonable separation performance and ionization yield 130 needs to be found. Liquid junction interfaces introduce a liquid 131 lined gap between the separation capillary and ionization source 132 that allows decoupling optimization of CE separation and ESI 133 parameters at low sample dilution effects. In-depth information about CE-MS technology, including glycan analysis using off- 134 line interfacing and further ionization techniques such as 135 matrix-assisted laser desorption/ionization (MALDI), can be 136 obtained from eminent recent review article $20.23-25$

Miniaturization of CE into microchann $Q$ levices offers 138 another attractive ESI-MS front end due to high sensitivity 139 analysis of nanoliter amounts of sample. ${ }^{26}$ Irrespective of the 140 chosen detection method, microchann ycan separations 141 generally offer ultra fast separation times heconds scale 142 but usually at the cost of resolution; particularly of lycans 143 carrying additional charged constituents, which are often 144 removed prior to analysis. ${ }^{27-29}$ For fast screening, e.g., of 145 $\mathrm{mAb}$ glycans during clone selection or rapid profiling of clinical 146 samples in disposable chips, where throughput is decisive, a 147 lower separation efficiency can be acceptable. Moreover, recent 148 introduction of longer separation channels has converged CE 149 and microchannel CE efficiencies but such designs yet need to 150 be commercialized. ${ }^{30-32}$

A dramatic gain in throughput is also possible by simply 152 running multiple separation columns in parallel. Multiplexed 153 systems are commercially available and incorporate from 4 up 154 to 96 capillaries. High experimental precision is maintained by 155 coinjection of internal standards for alignment. Multicapillary 156 systems are becoming increasingly established in clinical trials 157 and biomarker discovery studies, where hundreds of samples 158 are screened. ${ }^{33,34}$ However, commonly built-in electrokinetic 159 injection systems require careful sample handling, such as 160 maintaining consistent salt or free dye content. ${ }^{33}$ Avoiding 161 selective analyte injection is of utmost importance when peak 162 area based quantitation and associated statistical analyses are 163 conducted.

\section{GLYCAN STRUCTURE ELUCIDATION}

165

CE offers a variety of separation modes, which incorporate 166 different selectivity and associated glycan structure identifica- 167 tion potential. Counter-electroosmotic flow enables increasing 168 charge based migration grouping with respective larger species 169 migrating prior to smaller ones of the same net charge. ${ }^{36} 170$ Selectivity enhancement of structurally related glycan pools can 171 be achieved by online interaction with ionic buffer additives, 172 exhibiting different complexation affinity based upon mono- 173 saccharide composition. ${ }^{37}$ Zone electrophoresis in aqueous or 174 low viscosity polymer matrix with suppressed EOF provides 175 exceptionally high resolution of isomeric species due to the 176 separation principle of charge-to-hydrodynamic volume differ- 177 ences. ${ }^{38}$

Oligosaccharide Standards. Co-injection of purified 179 glycan standards with an unknown sample mixture poses a 180 simple and effective way for structural assignment. Although, 181 basic knowledge about the nature of the analyte pool present is 182 useful, because glycans with different monosaccharide or 183 linkage patterns can exhibit identical migration behavior. 184 Limited commercial availability of structures, their production 185 via purification with techniques offering restricted separation 186 efficiency (e.g., isomers), and taxonomy mismatch with the 187 sample further restrict practical implementation of standards. 188

Anticipation of molecular size from analyte migration time 189 can be achieved by the ancillary separation of gradually sized 190 oligomeric sugar standards. Optimal reference standards 191 incorporate equally distributed oligosaccharide pools exhibiting 192 a linear relationship between size and migration time. Thus, 193 linear homooligosaccharide ladders with degrees of polymer- 194 ization (DP) $1,2, \ldots, n$ of glucose $(\alpha 1 \rightarrow 4 \text { or } 6)_{n}$, glucose $(\beta 1 \rightarrow 195$ 

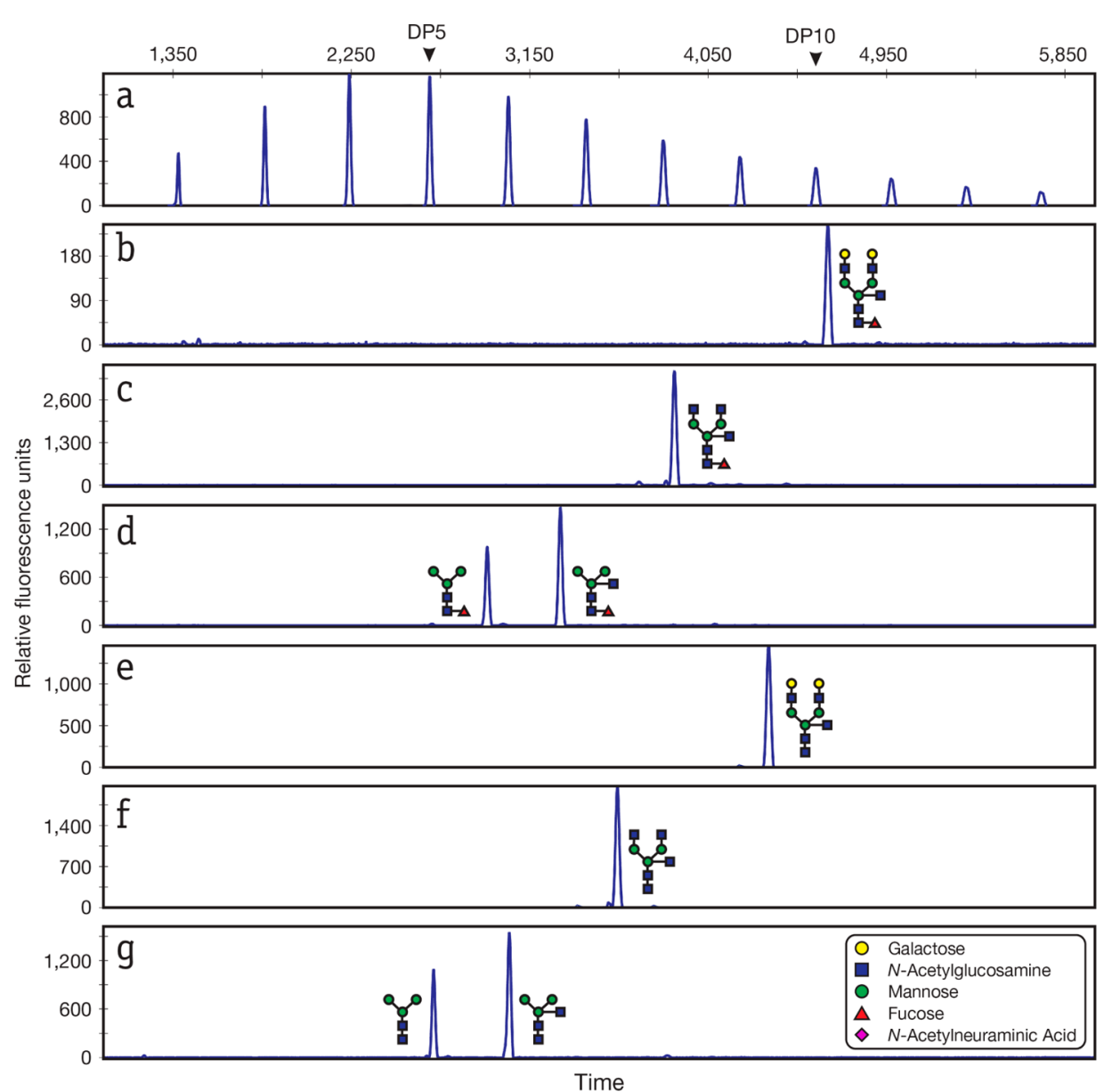

Figure 1. Exoglycosidase sequencing of a purified biantennary core fucosylated complex glycan standard with a bisecting $N$-acetylglucosamine. (a) Separation of an 8-aminopyrene-1,3,6-trisulphonic acid (APTS)-labeled oligomaltose hydrolysate reference standard. The number of glucose units (DP, degree of polymerization) in these structures is indicated. (b) Nondigested standard. Standard digested with (c) $\beta$-galactosidase, (d) $\beta$ - $N$ acetylhexosaminidase, and (e) $\alpha$-fucosidase. Standard digested simultaneously with (f) $\beta$-galactosidase and $\alpha$-fucosidase, (g) $\beta$-galactosidase, $\beta$ - $N$ acetylhexosaminidase, and $\alpha$-fucosidase. Symbols used for glycans are those suggested by the Consortium for Functional Glycomics. Reprinted with permission from ref 43. Copyright 2006 Nature Publishing Group.

$1964)_{n}$, and $N$-acetylglucosamine $(\beta 1 \rightarrow 4)_{n}$, produced by hydro197 lyzing starch, cellulose, and chitin-type polysaccharide chains, 198 respectively, are commonly applied standards. Hydrolysates of 199 branched structures, e.g., high-mannose type glycans, ${ }^{39}$ are 200 generally not recommended due to the occurrence of positional 201 isomers and the limited DP range. Molecular size standards can 202 either be coinjected with the sample, e.g., oligomer fragments of 203 DNA base pairs in multicapillary sequencer studies, ${ }^{40}$ or 204 analyzed in a separate experimental run, where alignment 205 standards are usually introduced in both sample and standard 206 runs. $^{41,42}$ Figure 1a,b shows the separation of a glucose207 oligomer ladder and a purified glycan standard, respectively. 208 The respective DP or sugar units (SU) of a sample peak can 209 be obtained by interpolation between adjacent oligosaccharide 210 ladder peaks or polynomial fitting of multiple standard peaks. ${ }^{44}$ 211 The conversion from migration time to a size-based scale of SU 212 also promotes interexperiment, -instrument, and -institution 213 precision and comparability, by compensating for potential 214 buffer composition, separation temperature, or column history 215 induced experimental deviations. The accumulation of SU 216 values of glycans with known (e.g., purified standards) or 217 decoded structural identity can evolve into the establishment of 218 a database, capable of decisively supporting structural 219 elucidation or denovo sequencing of unknown glycan pools.
Usage of such databases requires consistent experimental 220 conditions to those under which the data was generated, 221 including the appropriate ladder standard. Glucose units (GU), 222 i.e., SU based upon glucose oligomers, are widely used 223 normalization standards in glycan analysis. Maltooligosacchar- 224 ides $(\alpha 1 \rightarrow 4$ linked oligo-glucoses) are the prevalently used 225 standards in CE, whereas $\alpha 1 \rightarrow 6$ linked isomaltooligsacchar- 226 ides (also referred to as dextran) are predominantly applied in 227 glycan analysis by hydrophilic interaction liquid chromatog- 228 raphy (HILIC). Sole discrepancy in glycosidic linkage type or 229 anomericity of oligosaccharides can impact their hydrodynamic 230 volumes and result in differential migration, thus render SU 231 based structural assignments inaccurate if mismatching stand- 232 ards are used. ${ }^{44}$

Enzymatic Digests. The specific cleavage of monosacchar- 234 ide constituents from nonreducing termini via exoglycosidase 235 enzymatic digestion represents another powerful means for 236 glycan structure characterization. Depending on enzyme 237 specificity, monosaccharide type, sequence, or even linkage 238 and anomericity can be obtained when digest induced 239 structural/shape changes are accompanied by CE monitoring. 240 Sophisticated digest cascades can also lead to the identification 241 of positional isomers. ${ }^{45,46}$ Exoglycosidase digest arrays can 242 either be conducted in parallel, where the sample is evenly split 243 
244 into one aliquot per enzyme mixture, or in sequential fashion 245 using analysis-digest iterations of the same sample. Sequential 246 processing requires lower amounts of sample and enzymes and 247 takes advantage of the minimal injection volumes needed for $248 \mathrm{CE}$ analysis. The use of nonvolatile buffer components or 249 addition of alignment standards to the sample prior to 250 injection, as for example used in multicapillary sequencer 251 experiments, can impede further digestion steps. Moreover, 252 exoglycosidase digests can cause changes in the sample ionic 253 strength or $\mathrm{pH}$, introduced by salts of the digestion buffer, and 254 promote diffusion induced peak distortion as well as potentially 255 altered electrokinetic injection behavior. ${ }^{47}$ Volatile digestion 256 buffer systems are therefore generally recommended to alleviate 257 this issue. Most commercial enzymes are, however, supplied in 258 nonvolatile media and consequently will cause the described 259 problems in $\mathrm{CE}$, if concentrated sample volumes or multiple 260 enzymes are used.

261 Exoglycosidase digestion of glycans with known structure 262 (e.g., purified standards) can cause a predictable loss of 263 constituents corresponding to enzyme specificity. After 264 reanalyzing the digested product by $\mathrm{CE}$, glycan structural 265 differences can be recorded by the differences in migration time 266 and relative peak area compared to the substrate. Such shifts in 267 migration time, or respective sugar units, can be used to build a 268 knowledge base of monosaccharide residues and associated 269 "contributions". ${ }^{48}$ Traces $\mathrm{c}$ and $\mathrm{d}$ Q $\mathrm{e}$ and $\mathrm{g}$ in Figure 1 270 exemplify two different digest cascade possibilities of the 271 purified glycan standard analyzed in trace b. Structural identity 272 of hydrolyzed products, respective SU, as well as digest induced 273 shifts can readily be deduced. Additionally, positions of 274 unknowns, e.g., a monogalactosylated biantennary species, can 275 be anticipated half way between the bi- and the agalactosylated 276 peaks as, respectively, depicted in Figure 1b,c.

277 In the case of dealing with unknown glycan mixtures, 278 increasing structural identity confidence can be gained by 279 tracing respective peaks through several digestion steps or by 280 virtual "reattachment" of constituents in a bottom-up manner, 281 especially when combined with sugar unit shift analysis and CE282 based glycan structure reference databases. Relative peak areas 283 before and after digestion nevertheless need to be rationally 284 compared when analyzing complex oligosaccharide pools. 285 Glycans composed of different monosaccharide units can 286 exhibit identical migration, due to, e.g., similar charge to 287 hydrodynamic volume properties but differentially or even 288 unaffected enzymatic digest reactivity. This can be either due to 289 lacking the epitope that matches enzyme substrate specificity or 290 inaccessibility potentially induced by steric hindrance. For 291 example the removal of bisecting $N$-acetylglucosamine 292 (GlcNAc) residues upon hexosaminidase treatment can be 293 hampered as depicted in Figure 1d,g.

294 The hydrolysis of sialic acids, which introduces additional 295 molecular charges, by sialidase treatment prior to analysis, is a 296 rather regularly applied practice in CE based glycan 297 investigations. ${ }^{29,33,34,49-52}$ However, associating sialic acid 298 removal with technical limitations of electromigration based 299 methods is a misapprehension. For example, CZE with 300 suppressed EOF offered superior separation efficiency of 301 additionally charged thus faster migrating glycans, also when 302 compared with HILIC methods, due to decreased analyte 303 diffusion. ${ }^{53}$ The removal of sialic acids generally results in 304 decisive reduction of $\mathrm{CE}$ profile complexity, by merging 305 previously distributed corresponding species with a differential 306 degree of sialylation as well as sialic acid linkage and positional isomers. This reduction of complexity coincides with the loss of 307 information and potentially correlated physiological features, 308 when investigating glycans of biomedical interest. ${ }^{54,55} 309$ Although, the combination of previously distributed low 310 abundant species upon sialidase digestion might also allow 311 for the investigation of other biologically important glycan 312 features, formerly undetected due to dynamic range issues. 313 When electrokinetic injection is used, equalizing analyte charge 314 states will also support diminishing potentially biased injection. 315

Different endoglycosidases, used for the release of aspar- 316 agine-linked sugar moieties from glycoproteins, can be applied 317 for specific glycan type-based partitioning. While PNGase F 318 liberates all classes of $N$-linked glycans from mammalian 319 glycoproteins, Endo- $\beta$ - $N$-acetylglucosaminidase $\mathrm{H}$ (Endo $\mathrm{H}$ ) 320 specifically releases high-mannose and hybrid type oligosac- 321 charides. ${ }^{56}$ Endo $\mathrm{H}$ cleaves substrate glycans between the two 322 GlcNAc residues in the core region and readily enables the 323 identification of the specific glycan types by CE. ${ }^{57}$ Determi- 324 nation of the intersection and difference sets with the respective 325 PNGase F released pool enables type-based classification, ${ }^{58,59} 326$ when the alterations by the missing GlcNAc residue and 327 associated SU shifts are accounted for.

328

Exoglycosidase digests are usually carried out in low reaction 329 volumes $(\sim 10 \mu \mathrm{L})$ with overnight incubation to achieve 330 exhaustive enzymatic processing. On-column enzymatic 331 digestions represent a practic 12 id, low sample, and enzyme 332 consuming alternative with incubation times down to only a 333 few minutes or even during the separation process itself by 334 passing the injected sample through an enzyme plug. ${ }^{60}$ Direct 335 introduction of exoglycosidases into narrow bore separation 336 channels can increase enzymatic reaction speed due to 337 decreased diffusion limitations, but such endeavors are 338 sometimes incompatible with certain enzymes ${ }^{60}$ and often 339 accompanied by some loss in separation efficiency. ${ }^{51,61,62} 340$

Lectin Affinity. As opposed to consecutively cutting glycan 341 constituents, carbohydrate binding proteins, incorporated into 342 the separation column, can result in specific peak retardation or 343 disappearance, based upon their respective structural affinity. 344 Lectin affinity CE enables the structural classification of glycans 345 by type (e.g., high-mannose ${ }^{51,62,63}$ ), antennary branching (e.g., 346 triantennary ${ }^{64,65}$ ), monosaccharide features (e.g., fucose, ${ }^{60,65,66}{ }_{347}$ bisecting GlcNAc, ${ }^{64}$ galactose, $^{60}$ sialic acid ${ }^{65}$ ), or even by their 348 glycosidic linkage type (e.g., $\alpha 2-3$ or 2-6 linked sialic 349 acids ${ }^{60,67}$ ) via specific binding reactions. Similar to on-column 350 enzymatic digestions, lectins can either be added to the 351 $\mathrm{BGE}^{63-67}$ or introduced as a distinct zone, ${ }^{51,60,62}$ but generally 352 no incubation time is needed. Although, not all lectin- 353 carbohydrate interactions are well understood, binding 354 associations are generally low and peak distortion can limit 355 area based comparison of experiments with/without lectins, 356 affinity CE represents another fast and valuable structure 357 identification tool.

358

Additional Separation Dimensions. Additional orthogo- 359 nal separation dimensions offering different selectivity can 360 dra fic ly increase the resolution. Especially when investigating 361 the Iaentity of heterogeneous carbohydrate pools, the reduction 362 of sample complexity by preparative separation and subsequent 363 analysis of the collected fractions by CE is often indispensable. 364 Analytes can either be partitioned into groups of multiple 365 species according to sample properties such as charge state, or, 366 often by using several preparative techniques, into distinct 367 glycan species. The preparation of such purified carbchud ate 368 standards enables the identification strategies outline $\_$der 369 
370 Oligosaccharide Standards and Enzymatic Digests, when 371 combined with exoglycosidase digestion. For highly complex 372 samples consisting of a multitude of species, this approach can 373 be extremely labor-intensive or even impossible due to 374 restricted sample availability and resolution limitations of 375 preparative techniques in separating closely related structures. 376 Difficulties in structural identification arising from comigration 377 of species with similar charge to mass (or equivalent 378 hydrodynamic volume) ratios in $\mathrm{CE}$ can be minimized by 379 molecular charge or size based fractionation. Orthogonal 380 separation mechanisms, such as analyte polarity based surface 381 interaction, can also distinguish previously comigrating species, 382 due to differential separation selectivity.

383 Chromatographic techniques with various stationary phases 384 and corresponding distinct selectivities accompanied by higher 385 associated injection volume requirements are ideally suited for 386 preparative fractionation prior to $\mathrm{CE}$ analysis. Compatibility 387 with subsequent $\mathrm{CE}$ analysis is reached by desalting or the 388 application of volatile liquid phases. Similar to glycan analysis 389 by CE, sensitive detection in liquid chromatographic (LC) 390 techniques often requires the introduction of fluorescent dyes. 391 However, on the basis of the different selectivity of chromato392 graphic phases, difficulties can arise from certain properties of 393 labeling agents (e.g., charge, polarity) which were optimized for 394 glycan separation by CE. The combined application of 395 preparative LC and analytical CE techniques with sensitive 396 detection thus generally requires a compromise.

397 Anion-exchange chromatography (AEC) fractionation is one 398 of the popular means to separate glycan pools by their degree 399 of sialylation or other charge in ung modification (e.g., 400 phosphorylation). Each charged fracton may contain several 401 glycan structures, differing, e.g., in branching degree, 402 composition of monosaccharides, and their position or linkage 403 but all exhibiting the respective equal net negative charge. In 404 CZE under arheic conditions, for example, comigration of 405 larger structures holding higher charge states with smaller 406 analytes, can aggravate structural identification. Additional 407 charges introduced by sialic acids can be removed via sialidase 408 digestion and the resulting changes in relative peak areas 409 monitored, as outlined under Enzymatic Digests. In cases 410 where the ratio of charged vs neutral species is particularly 411 unbalanced in favor of the latter, e.g., sialo- vs asialo-glycans on 412 human IgG, and only trace amounts of certain charged species 413 are observed, exoglycosidase digestion induced increase of the 414 corresponding neutral species can be marginal. Partitioning into 415 charged fractions resolves overlaps between differentially 416 charged species and enables the monitoring of peak shifts to 417 unoccupied regions upon charge removal and direct compar418 ison to the associated neutral fraction. ${ }^{53}$

419 Figure 2 shows the high $\mathrm{pH}$ high performance AEC 420 (HPAEC) fractionation and consecutive $\mathrm{CE}$ analyses of 421 human serum $\mathrm{N}$-glycans released via endo $\mathrm{H}$. While sialidase 422 digest of the total pool in trace BS could result in inconclusive 423 changes of the corresponding neutral species, individual 424 analysis of preparatively captured fractions and their sialidase 425 digestions revealed the identity of the underlying monosialy426 lated hybrid type species, as presented in Figure $2 \mathrm{~B} .{ }^{57}$

427 Highly charged labeling agents, such as the commonly used 428 8-aminopyrene-1,3,6-trisulphonic acid (APTS), can critically 429 increase the total negative charge of analytes, thus potentially 430 causing excessive retention on AEC phases. Moreover, the 431 fundamental separation principle in AEC is based upon 432 differences in charge distribution across the hydrated molecule.
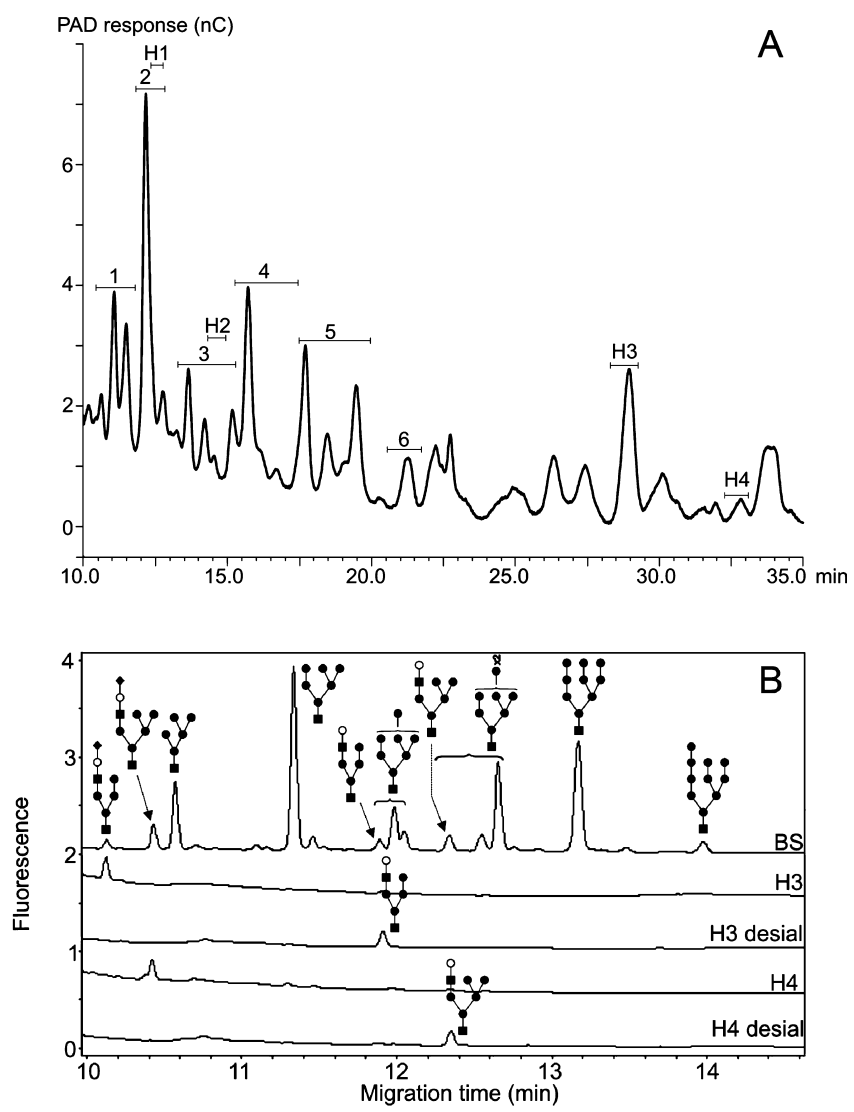

Figure 2. (A) HPAEC-PAD profile of human serum $N$-glycans released via endo $\mathrm{H}$. Areas subjected to fraction collection are indicated. (B) CE-laser induced fluorescence detection (LIF) trace of APTS labeled total glycan pool from blood serum (BS), collected fractions $\mathrm{H} 3, \mathrm{H} 4$ and their respective sialidase digests $(\mathrm{H} 3$ desial, $\mathrm{H} 4$ desial). Glycan symbols as in Figure 1. Adapted with permission from ref 57. Copyright 2011 John Wiley \& Sons, Inc.

The subdivision into distant non- and reducing terminal 433 charges further complicates the application of highly charged 434 dyes for AEC-based fractionation. Using APTS as an ionic dye 435 for $\mathrm{CE}$ separation and sensitive detection is still possible if 436 fractionation is carried out using underivatized glycans and only 437 subsequent dye conjugation. This was demonstrated by either 438 blind fraction collection adhering to pre-established retention 439 time windows from the separation of 2-aminobenzamide 440 labeled glycans ${ }^{53}$ or pulsed amperometric detection (PAD) 441 following HPAEC separation. ${ }^{57}$ On the basis of its separation 442 principle, AEC allowed for additional size-based separation 443 within each charged fraction, when operated with fluorescently 444 labeled glycans and sensitive optical detection. ${ }^{68}$ Supplemental 445 size-based fraction collection of underivatized glycans was also 446 achieved by UV detection ${ }^{69,70}$ or by HPAEC-PAD, which 447 enabled sensitive elution monitoring at maintaining adequate 448 resolution, as exemplarily shown in Figure 2A. HPAEC-based 449 separation generally requires a subsequent buffer exchange step 450 due to nonvolatile strongly alkaline mobile phases, the 451 described associated ionic strength mismatch issues in CE 452 separation, and high $\mathrm{pH}$-induced sample epimerizar ${ }_{453}^{1}$

Hydrophilic interaction liquid chromatograph 2 high 454 resolution fractionation alternative to AEC, also offering 455 orthogonal selectivity. Depending on the chromatographic 456 column, retention is a function of hydrophilicity and associated 457 glycan size or a combination with ionic interactions, ${ }^{72}$ when 458 

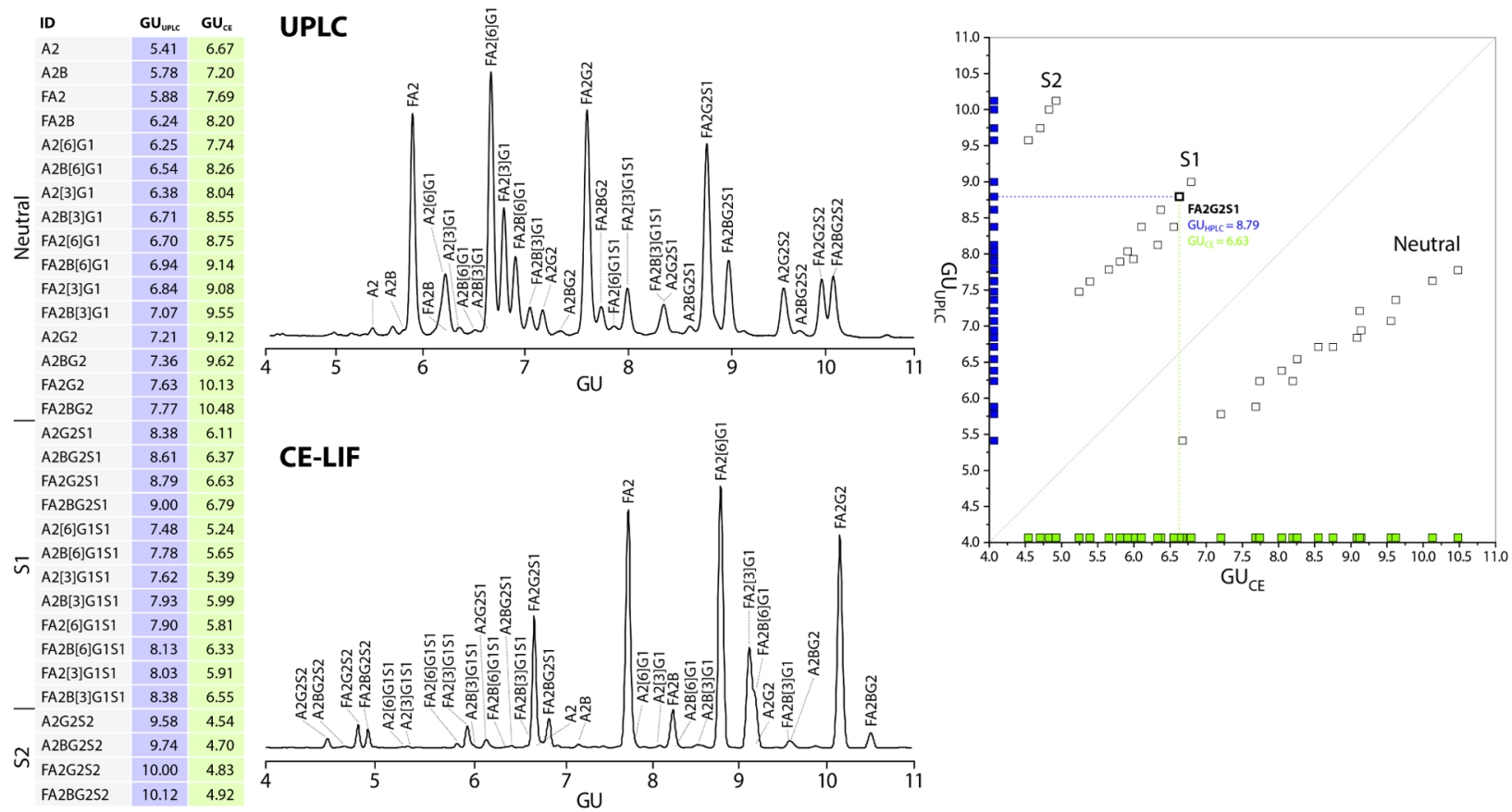

Figure 3. Comprehensive annotation of the UPLC-fluorescence and CE-LIF profiles of the N-glycans released from human serum polyclonal IgG using a combination of exoglycosidase digestion and weak anion exchange fractionation. Insets show a table of the experimentally determined GU value for each glycan structure and a comparative 2D plot. N-Glycan nomenclature from ref 91. Reprinted from ref 53. Copyright 2011 American Chemical Society.

459 using amide- or amine-based stationary phases, respectively. 460 HILIC-based separation enables the collection of fractions with 461 high individual structure purity as opposed to common analyte 462 property based grouping in AEC; however, the higher 463 resolution of the HILIC fractionation results in an increased 464 number of fractions and associated larger scale downstream 465 analysis. Combination of HILIC fractionation and exoglycosi466 dase digestion of purified analytes is also commonly applied for 467 accurate glycan structural elucidation. ${ }^{73-75}$

468 Similar to AEC, the application of polar and ionic labeling 469 agents, such as APTS (log $P-1.21)$ for CE analysis, was 470 preceded by either intact or removed charged glycan 471 constituents, using underivatized ${ }^{35}$ or APTS labeled oligosac472 charides, ${ }^{43,74}$ respectively. The motivations for these distinct 473 strategies, however, seem to originate from mass spectrometric 474 detection and $\mathrm{CE}$ instrument compatibility considerations. 475 Other fluorescent labeling agents, such as 2-aminobenzoic acid, 476 ( $\log P$ 0.78) featuring lower charge and polarity, were 477 successfully applied for both HILIC-based fractionation and $478 \mathrm{CE}$ analysis using high sensitivity fluorescence detection. ${ }^{73,76}$ 479 Applications of reverse phase (RP) and size-exclusion 480 chromatography (SEC) glycan fractionation are limited due 481 to lower associated applicability and selectivity, respectively, 482 when compared to HILIC and generally low efficiency for 483 relatively small sugars $(0.1-5 \mathrm{kDa}$ range), respectively. Only 484 few high performance columns serving ranges in the low 485 molecular weight region exist, ${ }^{77}$ and exclusive size-based 486 preparative partitioning on ion-exchange columns suffers from 487 low resolution. ${ }^{78}$ As opposed to HILIC, retention on RP 488 stationary phases is based on hydrophobicity thus providing 489 only weak interaction for polar glycans. Although, type based 490 separation of high mannose, complex and fucosylated complex 491 glycans can be achieved, ${ }^{79}$ and RP-based fractionation could 492 resolve comigration of species from the distinct groups. ${ }^{80}$ Also 493 the derivatization with more hydrophobic dyes, such as 2- aminoacridone (log $P$ 2.95), can facilitate $\mathrm{RP}$ fractionation 494 potential. $^{81}$

Capillary electrophoresis can also be operated in preparative 496 mode using larger capillary diameters and several interchange- 497 able outlet buffer reservoirs for fraction collection. ${ }^{82}$ Low 498 injection plug volumes often require several iterations of the 499 fractionation process to achieve a sufficient amount of analyte 500 per collected fraction. ${ }^{83}$ Micropreparative CE has nevertheless 501 been successfully applied for off-line MALDI ionization and 502 hyphenated mass detection, where higher concentration is 503 reached by liquid phase evaporation and concentration within 504 the MALDI matrix. ${ }^{84,85}$

One often perceived limitation of $\mathrm{CE}$ is the low sample 506 injection volume. However, this facilitates experiment repeti- 507 tion and also the consecutive execution of different CE 508 separation modes. Hence, the simplification of complex samples 509 via elaborate preparative fractionation can be avoided by the 510 introduction of additional analytical separation modes with 511 different selectivity. Glycans comigrating in one separation 512 dimension can be separated in another with appropriate 513 selectivity and vice versa. Such multidimensional mapping of 514 oligosaccharides originates from the application of different 515 stationary phases for liquid chromatography. ${ }^{86}$ Nanoliter 516 injection volumes and the versatility of $\mathrm{CE}$ allow the facile 517 operation of several different separation modes, including EOF 518 and on-column complexation phenomena, on the same 519 instrument or even capillary, by simple changing the electrolyte 520 used. Normalized migration times of identified carbohydrate 521 species from each separation dimension span a multidimen- 522 sional database thus facilitating structural identification of 523 unknowns at increasing confidence. ${ }^{87,88}$

Combination of analytical CE and LC glycan separation 525 methods comes at costs of additional instrumentation and 526 sample preparation steps, e.g., introduced by different 527 fluorescent labels optimized for each technique, but enables 528 

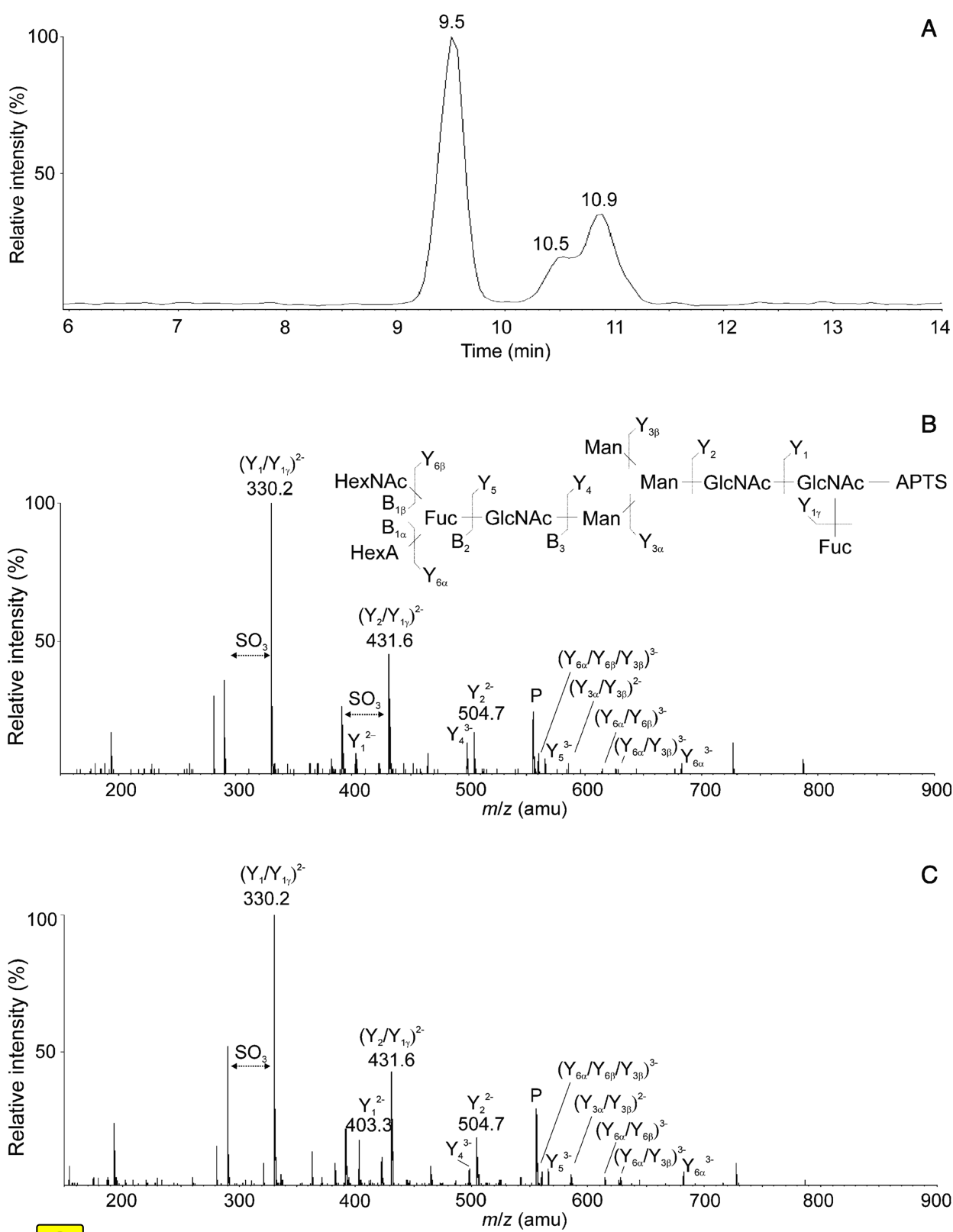

Figure 4. CE-MS/MS 2 f the fixed $m / z 555.7$ in Q1 of a quadrupole ion trap MS (A) and MS/MS fragmentation spectra of the respective peaks at 10.5 (B) and $10.9 \mathrm{~min}$ (C). The peak at 9.5 min corresponds to $\mathrm{Man}_{5} \mathrm{GlcNAc}_{2}$ at $m / z 557.8$. P, precursor ion. Fragmentation nomenclature as in ref 93. Reprinted with permission from ref 102. Copyright 2007 Oxford University Press.

529 orthogonal glycan separation. ${ }^{53,75}$ Although, fluorescent dyes 530 suitable for both platforms have been presented, ${ }^{73,76,89,90}$ 531 instrumental limitations such as available detection method, 532 can restrict their application. Disregarding the increased 533 necessary sample handling, the application of two distinct 534 analysis routes allows for monitoring potential experimental 535 discrepancies originating from, e.g., loss of labile glycan 536 constituents or salt content. Also one might not want to 537 change a certain analysis route due to already existing databases 538 that can be used for peak identification in the other 539 dimension. ${ }^{69}$

540 Figure 3 depicts the comprehensive annotation of the human 541 IgG N-glycan pool separated in both ultrahigh pressure LC 542 (UPLC)-HILIC and CE-LIF. Exhaustive glycan identification 543 was achieved by the combined application of preparative anion 544 exchange fractionation and exoglycosidase digestion steps requiring over $48 \mathrm{~h}$ of total analysis time. In a two-dimensional 545 plot, inset in Figure 3, all 32 oligosaccharides present were well 546 separated and revealed clustering based upon their degree of 547 sialylation. Combining high experimental precision, granted by 548 the use of glucose unit values following time based normal- 549 ization, with the beneficial orthogonality of the separation 550 platforms allowed for exhaustive and confident structural 551 annotation of the IgG N-glycan pool within only $20 \mathrm{~min}$ of 552 using the $2 \mathrm{D}$ space and made additional fractionation or 553 digestion techniques obsolete.

554

CE-MS: Molecular Mass and Fragmentation. Mass 555 spectrometry offers an information-rich complementary 556 detection method for glycan analysis, facilitating the measure- 557 ment of distinct analyte mass and charge properties when used 558 online, independent from standard coinjection or exoglycosi- 559 dase digest patterns. Glycan composition can be estimated 560 
561 based upon the addition of monosaccharide constituent masses. 562 However, because of the high structural diversity, numerous 563 combinations can result in equal mass values. Moreover, on the 564 monosaccharide level stereosiomers (e.g., glucose and man565 nose) exhibit the same masses, thus MS can only detect 566 different classes of sugars such as hexose (glucose, galactose, 567 mannose), $\mathrm{N}$-acetylhexosamine (GlcNAc or GalNAc), deoxy568 hexose (fucose), or sialic acids. Information about the identity 569 of monosaccharides may be deduced from additional sample 570 information such as glycan class (e.g., N-, O-glycan), taxonomy, 571 and associated biosynthetic pathways. Such information, if 572 available, provides very limited potential of elucidating linkage 573 and positional isobaric isomers. Therefore to increase the 574 overall level of information in each associated experiment, MS 575 detection can be combined with monosaccharide sequencing by 576 exoglycosidase digest induced mass shift monitoring or more 577 commonly the application of tandem mass spectrometry (MS/ $578 \mathrm{MS}$ ) following collision induced dissociation (CID). ${ }^{92}$ In 579 tandem MS or higher order $\mathrm{MS}^{n}$ approaches of pseudomo580 lecular oligosaccharide cations formed during positive ioniza581 tion, glycans first break at their most labile bonds resulting in a 582 series of glycosidic cleavages. Glycosidic linkage cleavage allows 583 determination of monosaccharide sequence and branching 584 degree, based on $\mathrm{B} / \mathrm{Y}$ or $\mathrm{C} / \mathrm{Z}$-ion series if negative ionization is 585 used, $^{93}$ whereas cross-ring cleavages and associated $\mathrm{A} / \mathrm{X}$-ions 586 allow deduction of linkage positions. ${ }^{92}$ Because of the 587 complexity and potential interpretation ambiguity, it is often 588 reasonable to investigate fragmentation patterns with purified 589 glycan standards similar to those under study. However, 590 informatics platforms such as GlycoWorkBench ${ }^{94}$ and Glyco591 PeakFinder ${ }^{95}$ have greatly helped in simplifying the inter592 pretation of oligosaccharide MS/MS spectra.

593 Problems arise from direct infusion of complex sample 594 mixtures, where selected precursor ion masses potentially 595 contain structural isomers. Fragmentation of multiple species 596 can lead to incorrect spectral interpretation and glycan mass 597 based structural conclusions. Hyphenation of MS with 598 separation based techniques can provide additional structural 599 information or even resolve isomeric species and thus prevent 600 erroneous structural interpretation from the resulting spectral 601 data. Concentration of complex analyte pools into distinct 602 bands using separation techniques results in increased dynamic 603 range and associated sensitivity for MS detection. Furthermore, 604 less analyte will enter the MS together and thereby reduce the 605 suppression of less abundant ions by predominant ones. Ion 606 suppression effects as well as biased quantitation of differ607 entially charged analytes and associated ionization yield can be 608 resolved by upstream CE separation due to the inherently high 609 efficiency of CE based separations.

610 MS interfacing generally comes at the compromise in CE 611 resolution, introduced by a MS friendly BGE, the missing outlet 612 buffer reservoir, differential ionization interface gas pressure, 613 siphoning effects, and band broadening in longer capillaries. On 614 the other hand, the more informative MS detection facilitates 615 the detection of comigrating nonisobaric analytes. High616 resolution off-line $\mathrm{CE}$ glycan separation with photometric 617 detection can be altered stepwise toward MS compatible 618 conditions. On the basis of relative abundances, respective 619 peaks can be assigned in the CE-MS base peak electrophero620 gram (BPE) and previously gathered structural information 621 matched or even amended. ${ }^{71,96,97}$ The more accurate 622 quantitation achieved by optical detection of heterogeneous analyte pools can also be incorporated online proximal to the 623 mass spectrometer. ${ }^{98-100}$

The separation of uncharged glycans in CE is problematic 625 because on-column complexation with borate or metal ions is 626 generally not MS compatible and sole EOF based separation 627 lacks selectivity. Isocratic elution in $\mathrm{CEC}^{21,101}$ or the 628 introduction of ionic labeling agents enable the parallel 629 separation of both neutral and charged species. Labels are 630 chosen not only based on CE separation optimization but also 631 MS ionization considerations. Neutral or positively charged 632 labeling agents, such as 9-fluorenylmethyl and 3-aminopyrazole, 633 are used for positive MS ionization polarity modes ${ }^{12,102}$ and 634 vice versa for the more commonly used APTS, 8-amino- 635 naphtalene-1,3,6-trisulfonic acid and 2-aminobenzoic acid in 636 the negative mode..$^{9,103,104}$

Figure 4A shows the CE-MS BPE of APTS labeled N- $638 \mathrm{f} 4$ glycans from the structural subunit 1 of Rapana venosa 639 hemocyanin at $\mathrm{m} / z 555.7$ isolated in Q1 of a quadrupole ion 640 trap MS. ${ }^{102}$ Counter-EOF conditions with the MS at the 641 cathodic side allowed separation based on increasing negative 642 charge and larger species migrating prior to smaller ones within 643 each charged group. The peak at 9.5 min corresponded to the 644 labeled $\mathrm{Man}_{5} \mathrm{GlcNAc}_{2}$ structure, whereas peaks at 10.5 and 10.9645 min represent isomers of a different glycan species with an 646 additional negative charge. Similar tandem MS spectra of the 647 peaks at 10.5 and 10.9, depicted in respective parts B and C of 648 Figure 4, endorsed matching the monosaccharide sequence and 649 suggested the presence of positional isomers that were 650 separated due to the high resolving power of CE.

651

Labeling at the reducing end can provide valuable 652 information on the site-attachment of specific glycan 653 constituents due to fragment ions either including or lacking 654 the mass and charge introduced by the labeling agent. A- and X- 655 type ions and associated linkage position elucidation, however, 656 are generally low when the reducing end of glycans is occupied, 657 because cross-ring fragmentation predominantly occurs at the 658 proximal end. ${ }^{105}$

Unlabeled glycans that carry charged constituents, such as 660 sialic acids or phosphate residues, were well separated in 661 counter-EOF CZE based upon their charge degree and 662 molecular size. ${ }^{106-109}$ Such acidic glycans are most sensitively 663 detected by negative ion MS, but positive mode allows 664 quantitation of differentially charged species, i.e., glycan pools 665 containing both positive and negative oligosaccharides, using, 666 e.g., acidic mobile phases in CEC that can protonate sialic 667 acids. ${ }^{101}$ Glycans with an unoccupied reducing end produce 668 more informative MS/MS spectra due to higher abundance of 669 $\mathrm{A} / \mathrm{X}$-ions, but reducing terminal mutarotation can increase 670 sample complexity. ${ }^{21}$ MS/MS fragmentation analysis of glycans 671 holding terminal sialic acids often leads to predominant $B_{1}$ ions 672 originating from the loss of such charged glycan constituents 673 and lower amounts of more informative C-type fragments. ${ }^{110} 674$

Chemical derivatization via amidation, methylation, or 675 permethylation can stabilize sialic acids ${ }^{111}$ and thus provide 676 greater structural elucidation potential in MS/MS fragmenta- 677 tion, ${ }^{112}$ but coinciding charge neutralization and increased 678 hydrophobicity limit their online CE-MS application. Offline 679 $\mathrm{CE}$ fraction collection and spotting on MALDI plates combines 680 CE efficiency with less complex MS spectra originated from 681 mostly singly charged ions..$^{84,85,113}$ Low mass loading in CE 682 limits chemical derivatization options of collected fractions. 683 These derivatizations are generally recommended to prevent 684 
685 glycan degradation during the higher energy laser desorption/ 686 ionization. $^{114}$

\section{CONCLUDING REMARKS AND PERSPECTIVES}

688 The pursuit of unrayning the glyco-puzzle progresses with the 689 evolution of high-re $Q$ ion separation techniques. Capillary 690 electrophoresis represents a rapid and high-resolution separa691 tion tool, which after sequencing the human genome found one 692 of its prime applications in the analysis of complex 693 carbohydrate mixtures. The ability of separating both linkage 694 and positional isomeric species based on inherent molecular 695 shape differences, such a 1 lrodynamic volume, has rendered $696 \mathrm{CE}$ indispensable for fin $\Omega$ uctural analysis. The underlying 697 separation principle can easily be influenced by capillary surface 698 modifications, replacing the background electrolyte, special 699 buffer additives, or ionic labeling agents to meet sample specific 700 optimal separation conditions. Despite the versatility of CE, the 701 introduction of additional separation dimensions should be 702 considered necessary to aid elucidating the vast structural 703 heterogeneity of glycans. Moreover, a parallel analysis route, 704 introducing, e.g., alternative labeling chemistry or orthogonal 705 separation, can prove beneficial for method validation and 706 obviating potential systematic bias. Application of comple707 mentary analytical approaches not only increases structural 708 identification accuracy but eventually opens up new possibilities 709 for the establishment of a score-based confidence system based 710 on data integration from the individual dimensions.

711 Sophisticated exoglycosidase digestion and mass spectro712 metric detection represent further powerful means to increase 713 structural elucidation confidence. Hyphenation of CE with MS 714 remains technically challenging but facilitates both sensitive and 715 highly informative detection when combined with the resolving 716 power of CE. MS also enables sensitive detection of unlabeled 717 glycans thus excluding potential associated analyte deterioration 718 or selective functionalization. Several informatics-based en719 deavors are being pursued to assist and (semi-) automate the 720 demanding interpretation of glycan $\mathrm{MS}$ and $\mathrm{MS} / \mathrm{MS}$ 721 fragmentation spectra.

722 Glycoinformatics tools to aid CE based glycan structure 723 elucidation still remain marginal. Extensive mapping databases 724 that enable structural inference from normalized d fedion 725 times of fluorescently derivatized glycans currently exist only 726 for HPLC based techniques and complementary databases for $727 \mathrm{CE}$ are necessitated. However, the immense structural diversity 728 of glycans, time-consuming experiments, and the limited 729 availability of glycan standards impede an exhaustive accumu730 lation of such data. As opposed to surface interaction based 731 separation techniques, glycan analysis results by CE could more 732 easily be predicted based on computed geometrical character733 istics and a plethora of structure database entries generated in 734 silico. The combination of the different data resources will 735 improve the confidence and quality of glycan structure 736 identification, for instance by $\mathrm{CE}$ separation based structure 737 constraints for subsequent MS/MS spectra annotation. There738 fore, it is anticipated that developing novel glycoinformatics 739 tools will play an equally important role as high-resolution 740 separation techniques and sensitive detection methods in 741 further re 10 ion of the glyco-puzzle.

\section{AUTHOR INFORMATION}

\section{Corresponding Author}

744 *E-mail: a.guttman@neu.edu. Phone: +36 (52) 411-717/ 745 64182. Fax: +36 (52) 255-539.
Notes

The authors declare no competing financial interest.

\section{ACKNOWLEDGMENTS}

This work was supported by the OTKA Grant K-81839 of the 749 Hungarian Research Council and the MTA-PE Translational 750 Glycomics Program (Grant 97101) of the Hungarian Academy 751 of Sciences. J.B. gratefully acknowledges funding from Science 752 Foundation Ireland under Grant Number 11/SIRG/B2107. 753

\section{REFERENCES}

(1) Mechref, Y.; Novotny, M. V. Chem. Rev. 2002, 102, 321-369. 755

(2) Huhn, C.; Ramautar, R.; Wuhrer, M.; Somsen, G. W. Anal. 756 Bioanal. Chem. 2010, 396, 297-314.

(3) Chiesa, C. Horvath C. J. Chromatogr. 1993, 645, 337-352.

(4) Camilleri, P.; Harland, G. B.; Okafo, G. Anal. Biochem. 1995, 230, 759 $115-122$.

(5) Klockow, A.; Amado, R. Widmer, H. M.; Paulus, A. 76 Electrophoresis 1996, 17, 110-119.

(6) Charlwood, J.; Birrell, H.; Organ, A.; Camilleri, P. Rapid 763 Commun. Mass Spectrom. 1999, 13, 716-723.

(7) Harland, G. B.; Okafo, G.; Matejtschuk, P.; Sellick, I. C.; 765 Chapman, G. E.; Camilleri, P. Electrophoresis 1996, 17, 406-411. 766

(8) Honda, S.; Okeda, J.; Iwanaga, H.; Kawakami, S.; Taga, A.; 767 Suzuki, S.; Imai, K. Anal. Biochem. 2000, 286, 99-111. 768

(9) Tran, N. T.; Taverna, M.; Deschamps, F. S.; Morin, P.; Ferrier, D. 769 Electrophoresis 1998, 19, 2630-2638.

(10) Zhong, H.; El Rassi, Z. J. Sep. Sci. 2009, 32, 10-20.

(11) Guttman, A. Nature (London) 1996, 380, 461-462. 772

(12) Nakano, M.; Higo, D.; Arai, E.; Nakagawa, T.; Kakehi, K.; 773 Taniguchi, N.; Kondo, A. Glycobiology 2009, 19, 135-143. 774

(13) Oyama, T.; Yodohsi, M.; Yamane, A.; Kakehi, K.; Hayakawa, T.; 775 Suzuki, S. J. Chromatogr., B: Anal. Technol. Biomed. Life Sci. 2011, 879, 776 2928-2934.

(14) Anumula, K. R. Anal. Biochem. 2006, 350, 1-23. 778

(15) Ruhaak, L. R.; Zauner, G.; Huhn, C.; Bruggink, C.; Deelder, A. 779 M.; Wuhrer, M. Anal. Bioanal. Chem. 2010, 397, 3457-3481. 780

(16) Harvey, D. J. J. Chromatogr., B: Anal. Technol. Biomed. Life Sci. 781 2011, 879, 1196-1225.

(17) Hara, S.; Yamaguchi, M.; Takemori, Y.; Nakamura, M.; Ohkura, 783 Y. J. Chromatogr., Biomed. Appl. 1986, 377, 111-119. 784

(18) Zanetta, J.-P.; Pons, A.; Iwersen, M.; Mariller, C.; Leroy, Y.; 785 Timmerman, P.; Schauer, R. Glycobiology 2001, 11, 663-676. 786

(19) Honda, S. J. Chromatogr., A 1996, 720, 337-351.

(20) Pioch, M.; Bunz, S.-C.; Neusuess, C. Electrophoresis 2012, 33, 788 $1517-1530$

(21) Que, A. H.; Novotny, M. V. Anal. Bioanal. Chem. 2003, 375, 790 $599-608$.

(22) Yoshino, K.-i.; Takao, T.; Murata, H.; Shimonishi, Y. Anal. 792 Chem. 1995, 67, 4028-4031.

(23) Krenkova, J.; Foret, F. Proteomics 2012, 12, 2978-2990.

(24) Mechref, Y.; Novotny, M. V. Mass Spectrom. Rev. 2009, 28, 795 207-222.

(25) Zhao, S. S.; Zhong, X.; Tie, C.; Chen, D. D. Y. Proteomics 2012, 797 12, 2991-3012.

(26) Bindila, L.; Peter-Katalinic, J. Mass Spectrom. Rev. 2009, 28, 799 223-253.

(27) Dang, F.; Kakehi, K.; Nakajima, K.; Shinohara, Y.; Ishikawa, M.; 801 Kaji, N.; Tokeshi, M.; Baba, Y. J. Chromatogr., A 2006, 1109, 138-143. 802

(28) Primack, J.; Flynn, G. C.; Pan, H. Electrophoresis 2011, 32, 803 $1129-1132$.

804

(29) Vanderschaeghe, D.; Szekrenyes, A.; Wenz, C.; Gassmann, M.; 805 Naik, N.; Bynum, M.; Yin, H.; Delanghe, J.; Guttman, A.; Callewaert, 806 N. Anal. Chem. 2010, 82, 7408-7415.

(30) Mitra, I.; Zhuang, Z.; Zhang, Y.; Yu, C.-Y.; Hammoud, Z. T.; 808 Tang, H.; Mechref, Y.; Jacobson, S. C. Anal. Chem. 2012, 84, 3621- 809 3627. 
811 (31) Zhuang, Z.; Starkey, J. A.; Mechref, Y.; Novotny, M. V.; 812 Jacobson, S. C. Anal. Chem. 2007, 79, 7170-7175.

813 (32) Zhuang, Z.-X.; Mitra, I.; Hussein, A.; Novotny, M. V.; Mechref, 814 Y.; Jacobson, S. C. Electrophoresis 2011, 32, 246-253.

815 (33) Fang, M.; Zhao, Y.-P.; Zhou, F.-G.; Lu, L.-G.; Qi, P.; Wang, H.; 816 Zhou, K.; Sun, S.-H.; Chen, C.-Y.; Gao, C.-F. Int. J. Cancer 2010, 127, 817 148-159.

818 (34) Vanderschaeghe, D.; Laroy, W.; Sablon, E.; Halfon, P.; Van 819 Hecke, A.; Delanghe, J.; Callewaert, N. Mol. Cell. Proteomics 2009, 8, 820 986-994.

821 (35) Ruhaak, L. R.; Hennig, R.; Huhn, C.; Borowiak, M.; Dolhain, R. 822 J. E. M.; Deelder, A. M.; Rapp, E.; Wuhrer, M. J. Proteome Res. 2010, 9, 823 6655-6664.

824 (36) Hermentin, P.; Doenges, R.; Witzel, R.; Hokke, C. H.; 825 Vliegenthart, J. F. G.; Kamerling, J. P.; Conradt, H. S.; Nimtz, M.; 826 Brazel, D. Anal. Biochem. 1994, 221, 29-41.

827 (37) Szabo, Z.; Guttman, A.; Bones, J.; Karger, B. L. Anal. Chem. $8282011,83,5329-5336$.

829 (38) Guttman, A.; Pritchett, T. Electrophoresis 1995, 16, 1906-1911. 830 (39) Wolff, M. W.; Murhammer, D. W.; Linhardt, R. J. Prep. Biochem. 831 Biotechnol. 1999, 29, 1-21.

832 (40) Schwarzer, J.; Rapp, E.; Reichl, U. Electrophoresis 2008, 29, 833 4203-4214.

834 (41) Suzuki, S.; Honda, S. TrAC, Trends Anal. Chem. 1995, 14, 279835288.

836 (42) Nashabeh, W.; El Rassi, Z. J. Chromatogr. 1992, 600, 279-287. 837 (43) Laroy, W.; Contreras, R.; Callewaert, N. Nat. Protoc. 2006, 1, 838 397-405.

839 (44) Mittermayr, S.; Guttman, A. Electrophoresis 2012, 33, 10008401007.

841 (45) Ma, S.; Nashabeh, W. Anal. Chem. 1999, 71, 5185-5192.

842 (46) Szabo, Z.; Guttman, A.; Bones, J.; Shand, R. L.; Meh, D.; Karger, 843 B. L. Mol. Pharm. 2012, 9, 1612-1619.

844 (47) Mikkers, F. E. P.; Everaerts, F. M.; Verheggen, T. P. E. M. J. 845 Chromatogr. 1979, 169, 1-10.

846 (48) Guttman, A. Electrophoresis 1997, 18, 1136-1141.

847 (49) Callewaert, N.; Van Vlierberghe, H.; Van Hecke, A.; Laroy, W.; 848 Delanghe, J.; Contreras, R. Nat. Med. 2004, 10, 429-434.

849 (50) Dang, F.; Kakehi, K.; Cheng, J.; Tabata, O.; Kurokawa, M.; 850 Nakajima, K.; Ishikawa, M.; Baba, Y. Anal. Chem. 2006, 78, 14528511458 .

852 (51) Luo, R.; Archer-Hartmann, S. A.; Holland, L. A. Anal. Chem. $8532010,82,1228-1233$.

854 (52) Zhao, Y.-P.; Ruan, C.-P.; Wang, H.; Hu, Z.-Q.; Fang, M.; Gu, X.; 855 Ji, J.; Zhao, J.-Y.; Gao, C.-F. Cancer 2012, 118, 639-650.

856 (53) Mittermayr, S.; Bones, J.; Doherty, M.; Guttman, A.; Rudd, P. 857 M. J. Proteome Res. 2011, 10, 3820-3829.

858 (54) Adamczyk, B.; Tharmalingam, T.; Rudd, P. M. Biochim. Biophys. 859 Acta, Gen. Subj. 2012, 1820, 1347-1353.

860 (55) Varki, A. Trends Mol. Med. 2008, 14, 351-360.

861 (56) O’Neill, R. A. J. Chromatogr., A 1996, 720, 201-215.

862 (57) Frisch, E.; Kaup, M.; Egerer, K.; Weimann, A.; Tauber, R.; 863 Berger, M.; Blanchard, V. Electrophoresis 2011, 32, 3510-3515.

864 (58) Fenaille, F.; Le Mignon, M.; Groseil, C.; Ramon, C.; Riande, S.; 865 Siret, L.; Bihoreau, N. Glycobiology 2007, 17, 932-944.

866 (59) Kakehi, K.; Funakubo, T.; Suzuki, S.; Oda, Y.; Kitada, Y. J. 867 Chromatogr., A 1999, 863, 205-218.

868 (60) Yagi, Y.; Yamamoto, S.; Kakehi, K.; Hayakawa, T.; Ohyama, Y.; 869 Suzuki, S. Electrophoresis 2011, 32, 2979-2985.

870 (61) Archer-Hartmann, S. A.; Crihfield, C. L.; Holland, L. A. 871 Electrophoresis 2011, 32, 3491-3498.

872 (62) Archer-Hartmann, S. A.; Sargent, L. M.; Lowry, D. T.; Holland, 873 L. A. Anal. Chem. 2011, 83, 2740-2747.

874 (63) Hong, M.; Cassely, A.; Mechref, Y.; Novotny, M. V. J. 875 Chromatogr., B: Biomed. Sci. Appl. 2001, 752, 207-216.

876 (64) Nakajima, K.; Oda, Y.; Kinoshita, M.; Kakehi, K. J. Proteome Res. 877 2003, 2, 81-88.

878 (65) Nakajima, K.; Kinoshita, M.; Oda, Y.; Masuko, T.; Kaku, H.; 879 Shibuya, N.; Kakehi, K. Glycobiology 2004, 14, 793-804.
(66) Dang, F.; Maeda, E.; Osafune, T.; Nakajima, K.; Kakehi, K.; 880 Ishikawa, M.; Baba, Y. Anal. Chem. 2009, 81, 10055-10060. 881

(67) Ishizuka, A.; Hashimto, Y.; Naka, R.; Kinoshita, M.; Kakehi, K.; 882 Seino, J.; Funakoshi, Y.; Suzuki, T.; Kameyama, A.; Narimatsu, H. 883 Biochem. J. 2008, 413, 227-237. 884

(68) Bones, J.; McLoughlin, N.; Hilliard, M.; Wynne, K.; Karger, B. 885 L.; Rudd, P. M. Anal. Chem. 2011, 83, 4154-4162. 886

(69) Hermentin, P.; Witzel, R.; Doenges, R.; Bauer, R.; Haupt, H.; 887 Patel, T.; Parekh, R. B.; Brazel, D. Anal. Biochem. 1992, 206, 419-429. 888

(70) Taverna, M.; Baillet, A.; Biou, D.; Schluter, M.; Werner, R.; 889 Ferrier, D. Electrophoresis 1992, 13, 359-366.

(71) Liu, Y.; Salas-Solano, O.; Gennaro, L. A. Anal. Chem. 2009, 81, 891 $6823-6829$.

(72) Suzuki, S.; Tanaka, R.; Takada, K.; Inoue, N.; Yashima, Y.; 893 Honda, A.; Honda, S. J. Chromatogr., A 2001, 910, 319-329.

(73) Kamoda, S.; Ishikawa, R.; Kakehi, K. J. Chromatogr., A 2006, 895 1133, 332-339.

(74) Liu, X.-E.; Desmyter, L.; Gao, C.-F.; Laroy, W.; Dewaele, S.; 897 Vanhooren, V.; Wang, L.; Zhuang, H.; Callewaert, N.; Libert, C.; 898 Contreras, R.; Chen, C. Hepatology 2007, 46, 1426-1435.

(75) Quintero, O.; Montesino, R.; Cremata, J. A. Anal. Biochem. 900 1998, 256, 23-32.

(76) Maeda, E.; Kita, S.; Kinoshita, M.; Urakami, K.; Hayakawa, T.; 902 Kakehi, K. Anal. Chem. 2012, 84, 2373-2379.

(77) Shi, X.; Zaia, J. J. Biol. Chem. 2009, 284, 11806-11814. 904

(78) Honda, S.; Makino, A.; Suzuki, S.; Kakehi, K. Anal. Biochem. 905 1990, 191, 228-234.

(79) Chen, X.; Flynn, G. C. Anal. Biochem. 2007, 370, 147-161. 907

(80) Prater, B. D.; Connelly, H. M.; Qin, Q.; Cockrill, S. L. Anal. 908 Biochem. 2009, 385, 69-79.

(81) Okafo, G.; Burrow, L.; Carr, S. A.; Roberts, G. D.; Johnson, W.; 910 Camilleri, P. Anal. Chem. 1996, 68, 4424-4430.

(82) Guttman, A.; Sperling, E.; Mazsaroff, I. J. Liq. Chromatogr. Relat. 912 Technol. 1996, 19, 1539-1549.

(83) Wolff, M. W.; Bazin, H. G.; Lindhardt, R. J. Biotechnol. Tech. 914 1999, 13, 797-801.

(84) An, H. J.; Franz, A. H.; Lebrilla, C. B. J. Chromatogr., A 2003, 916 $1004,121-129$.

(85) Suzuki, H.; Mueller, O.; Guttman, A.; Karger, B. L. Anal. Chem. 918 1997, 69, 4554-4559.

(86) Tomiya, N.; Awaya, J.; Kurono, M.; Endo, S.; Arata, Y.; 920 Takahashi, N. Anal. Biochem. 1988, 171, 73-90. (87) Suzuki, S.; Kakehi, K.; Honda, S. Anal. Biochem. 1992, 205, 922 227-236.

(88) Zieske, L. R.; Fu, D.; Khan, S. H.; O’Neill, R. A. J. Chromatogr., 924 A 1996, 720, 395-407.

(89) Briggs, J. B.; Keck, R. G.; Ma, S.; Lau, W.; Jones, A. J. S. Anal. 926 Biochem. 2009, 389, 40-51.

(90) Ruhaak, L. R.; Huhn, C.; Waterreus, W.-J.; de Boer, A. R.; 928 Neusuess, C.; Hokke, C. H.; Deelder, A. M.; Wuhrer, M. Anal. Chem. 929 2008, 80, 6119-6126.

(91) Harvey, D. J.; Merry, A. H.; Royle, L.; Campbell, M. P.; Dwek, 931 R. A.; Rudd, P. M. Proteomics 2009, 9, 3796-3801.

(92) Zaia, J. Chem. Biol. 2008, 15, 881-892.

(93) Domon, B.; Costello, C. E. Glycoconjugate J. 1988, 5, 397-409. 934

(94) Ceroni, A.; Maass, K.; Geyer, H.; Geyer, R.; Dell, A.; Haslam, S. 935 M. J. Proteome Res. 2008, 7, 1650-1659. 936

(95) Maass, K.; Ranzinger, R.; Geyer, H.; von der Lieth, C.-W.; 937 Geyer, R. Proteomics 2007, 7, 4435-4444. 938

(96) Gennaro, L. A.; Salas-Solano, O.; Ma, S. Anal. Biochem. 2006, 939 $355,249-258$.

(97) Jayo, R. G.; Li, J.; Chen, D. D. Y. Anal. Chem. 2012, 84, 8756- 941 8762.

(98) Albrecht, S.; Schols, H. A.; van den Heuvel, E. G. H. M.; 943 Voragen, A. G. J.; Gruppen, H. Electrophoresis 2010, 31, 1264-1273. 944 (99) Gennaro, L. A.; Salas-Solano, O. Anal. Chem. 2008, 80, 3838- 945 3845. 
947 (100) Huhn, C.; Ruhaak, L. R; Mannhardt, J.; Wuhrer, M.; 948 Neusuess, C.; Deelder, A. M.; Meyer, H. Electrophoresis 2012, 33, $949563-566$.

950 (101) Que, A. H.; Mechref, Y.; Huang, Y.; Taraszka, J. A.; Clemmer, 951 D. E.; Novotny, M. V. Anal. Chem. 2003, 75, 1684-1690.

952 (102) Sandra, K.; Dolashka-Angelova, P.; Devreese, B.; Van 953 Beeumen, J. Glycobiology 2007, 17, 141-156.

954 (103) Gennaro, L. A.; Delaney, J.; Vouros, P.; Harvey, D. J.; Domon, 955 B. Rapid Commun. Mass Spectrom. 2002, 16, 192-200.

956 (104) Sandra, K.; Van Beeumen, J.; Stals, I.; Sandra, P.; Claeyssens, 957 M.; Devreese, B. Anal. Chem. 2004, 76, 5878-5886.

958 (105) Harvey, D. J. J. Am. Soc. Mass Spectrom. 2000, 11, 900-915.

959 (106) Balaguer, E.; Demelbauer, U.; Pelzing, M.; Sanz-Nebot, V.; 960 Barbosa, J.; Neususs, C. Electrophoresis 2006, 27, 2638-2650.

961 (107) Balaguer, E.; Neusuess, C. Anal. Chem. 2006, 78, 5384-5393.

962 (108) Ongay, S.; Martin-Alvarez, P. J.; Neususs, C.; de Frutos, M. 963 Electrophoresis 2010, 31, 3314-3325.

964 (109) Ongay, S.; Neusuess, C. Anal. Bioanal. Chem. 2010, 398, 845965855.

966 (110) Leymarie, N.; Zaia, J. Anal. Chem. 2012, 84, 3040-3048.

967 (111) Wheeler, S. F.; Domann, P.; Harvey, D. J. Rapid Commun. 968 Mass Spectrom. 2009, 23, 303-312.

969 (112) Ashline, D.; Singh, S.; Hanneman, A.; Reinhold, V. Anal. Chem. $9702005,77,6250-6262$.

971 (113) Tegeler, T. J.; Mechref, Y.; Boraas, K.; Reilly, J. P.; Novotny, 972 M. V. Anal. Chem. 2004, 76, 6698-6706.

973 (114) Kang, P.; Mechref, Y.; Klouckova, I.; Novotny, M. V. Rapid 974 Commun. Mass Spectrom. 2005, 19, 3421-3428. 